\section{Zierliche Mutter Courage oder: "a life in a nutshell»}

\author{
A. Hazan
}

Die zierliche kleine Patientin, sechsundachtzigjährig, mit medizinballgrossem Bauch - "marron sur deux allumettes?» - wird vom Kollegen in der Gemeinschaftspraxis zur gynäkologischen Untersuchung geschickt. Sie hat lebhafte, liebe, kleine Augen, das rechte ist eingesunken. Sie atmet etwas schwer, das sei neu, noch vor nicht so langer Zeit sei sie mit ihren Söhnen auf Wanderungen gegangen, deshalb habe sie sich ja auch - erstmals mit 80 Jahren - die Kniegelenke noch operieren lassen. Ich helfe ihr beim Ausdem-Mieder-Steigen: "Ich bi schono froh, dass Sie ä Frau sind - bim nä Maa het ich mich dänn schön geniert!» Die letzte gynäkologische Kontrolle, ja, die habe sie vielleicht vor etwa dreissig Jahren gehabt, damals beim Jelmoli, da habe man vom Personalarzt einen Gutschein bekommen für den Untersuch beim Frauenarzt, den musste man unterschrieben zurückbringen. Was sie denn beruflich gemacht habe - «Ja, ebä nüt! Ich bi Verchäuferin gsi, 25 Jahr lang bim Jelmoli - si händ mi ja damals nöd wellä, d'Schwiizer. Mini Mueter hät halt än Dütschä ghüratet, aber min Vater isch grad am Afang vom 1. Wältchrieg umcho, und ich bi dänn mit minere Mueter mit elfi zrugg i'd Schwyz cho, aber si händ mi nöd anärkannt, und ich ha nöd dörfä än Bruäf lernä. Dänn han i spöter no müessä än Ariernachwies liferä. Debi han i doch kei Ahnig gha vo dä Sitä vo mim Vater. Erscht won i ghüratä han, da bin i Schwyzeri wordä.» Sie steigt etwas mühselig auf den Gynistuhl. Ich erkläre ihr nochmals kurz, was ich machen werde. Sie fragt, ob es denn Krebs sein könnte - ja, wo der Doktor von Metastasen in der Lunge gesprochen habe, habe sie natürlich schon gewusst, "was äs gschället hät». Aber sie habe keine Angst vor dem Tod, es sei ja etwas anderes, wenn eine junge Mutter von Kindern wegstürbe. Sie habe ja ihr Leben gehabt, und ihre Söhne seien jetzt Gott sei Dank erwachsen und hätten nette Familien. Ja, ganz allein habe sie sie aufgezogen, schon seit die Söhne ein und drei Jahre alt waren, sei sie mit ihnen allein gewesen - «min Maa hät halt dänn scho wider än andäri gha». Aber schön hätten sie es gehabt, zwar sei’s streng gewesen, aber über's Wochenende seien sie immer auf schöne Wanderungen gegangen. "Und öppis Rächts isch doch au usänä gwordä, der eint isch in Amerika gsi und isch jetzt Leiter vomänä Finanzistitut i dä Schwiiz, dä ander hät

Korrespondenz:

Andrea Hazan, med. pract.

Guggachstrasse 34

CH-8057 Zürich es eigenes Gschäft mit so Pumpsystem und Wärmeregler." Ich schaue indes durch's Spekulum: atrophe Vagina und Cervix, blutet sogleich, Cervix höckerig, merkwürdig, dieser gelbe Fluor. "Won i vor zwei Jahr d'Chnü normal ha la operiere, da hät dä Chefchirurg mir wellä dä Herd us dä Lungä usäholä. Aber ich han im gseit, mer heg än doch scho vor vier Jahr gse, woni ha müessä Huut verpflanzä la, und er seg ja nöd fescht gwachsä sid döt, und ich welli i mim Alter sicher dä nümmä operierä. Ich han äm grad ä chli wüescht müessä säge, aber er hät's dänn verstandä. Wüssäd $\mathrm{Si}$, ich bin ja vil Chranki go bsuächä - mängi sind aber so schlächt dra gsi, mit offenäm Mul daglägä, mä hät si immer wider müessä cheerä, wi läbigi Liichnam - nei, so wett i dänn scho nöd sterbä. Ich ha drum ganz dütlich scho vor Jahrä gschribä, dass ich nöd wot künschtlich am Läbä ghalte werdä, eifach liidä möchti nöd - möglichscht schmerzfrei und schnäll vo derä Wält abträtä, das wünsch ich mir.» Die PAP-Entnahme ist fertig, die Palpation bei dem gespannten, aufgedunsenen Abdomen nicht gerade aussagekräftig, rektal frei. Jetzt käme noch der Vaginal-US. Die Patientin muss aber dringend Wasser lösen, obschon sie gerade eben erst war. Ja, das sei in letzter Zeit ein Problem, ob da wohl auch der grosse Bauch drücke? Der sei in den letzten Wochen immer grösser geworden, dabei esse sie doch so wenig und habe auch nicht an Gewicht zugenommen. Auch das Erbrechen am Morgen, sobald sie aufstehe, habe mit dem Bauch angefangen.

So rasch als möglich wird Mieder und das Nötigste wieder angezogen und auf die Toilette pressiert. Erleichtert kommt sie zurück. In einem anderen Fall wäre ich jetzt wohl vom Zeitdruck gehetzt worden, nicht so mit dieser Patientin: ich hatte ein fast respektvolles Gefühl, dass diese Zeit mit ihr wichtig, einmalig sei.

Nochmals das Ausziehritual. Man müsse ja auch die Kosten im Gesundheitswesen nicht noch mehr herauftreiben und unnötige Operationen machen, nimmt sie den Faden wieder auf. Ich werfe ein, dass sie es jetzt ja aber sicher zugute habe, sie habe sicher nie zuviel gehabt, und man sollte doch wenigstens herausfinden, was los sei, damit ihr gerade so, wie sie es sich wünsche, noch geholfen werden könne. Ja, für sich selbst habe sie nie viel beansprucht, auch jetzt, wie sollte sie auch grosse Sprünge machen, sie habe ja nur die AHV. Aber ihre Söhne sorgten schon für sie. Im Schall ein kleiner Uterus, Flüssigkeit im Douglas und eine diffuse Echogenizität darüber, die ich nicht interpretieren kann. Ja, doch, sie würde am Nachmittag schon noch zum andern Kollegen in der Praxis kommen, für einen abdominalen Ultraschall. $\mathrm{Ob}$ es denn nun Krebs sei? Ich sage, es sehe schon nach so etwas aus. Vielleicht wäre es besser, alles im Spital noch genauer abzuklären. Wie sie dies bisher gemacht habe, solle sie auch weiterhin genau nachfragen, was wozu gemacht werde und darüber bestimmen, ob sie dies auch möchte. Ja, das werde sie. Einmal komme eben die Zeit zu sterben, und in ihrem Alter sei dies ja zu erwarten. Sie habe ihr Leben gelebt, habe es doch gut mit ihren Söhnen gehabt, sie 
könne jetzt gut gehen. Einfach leiden wolle sie nicht. Sie zieht sich wieder an.

Ich bin auf eine Art betroffen, Ehrfurcht hatte mir soeben auf die Schulter getippt: ob ich im Alter, sollte ich es denn erreichen, auch einmal so heiter und insgesamt zufrieden mit mir würde sterben können, wo ich doch schon jetzt, trotz unvergleichlich viel leichteren Bedingungen immer wieder mit dem Leben und mir selbst haderte. Die liebe Frau, ihren schweren Bauch schiebend und atmend, verabschiedet sich warmherzig und würdig, eine ganz spezielle Persönlichkeit und geistig blitzklar.

Ich musste dieses Wochenende viel an sie denken, überlegte mir, über sie zu schreiben, nicht wissend, dass es ein Nachruf werden würde. Der Kollege hatte sie gleichentags nach dem Ultraschall, wo multiple Lebermetastasen und massiver Aszites gefunden wurden, für eine palliative, abklärende Kurzhospitalisation einweisen wollen, das war Montag. Die Patientin wollte noch einiges erledigen und erst eine Woche später eintreten. Am Wochenende ist sie dann wegen der Dyspnoe selbst ins Spital gegangen, sie habe noch beim Eintrittsuntersuch mit den Ärzten gescherzt. Man dachte bei der Dyspnoe auch an multiple kleinere Lungenembolien, da der abpunktierte Liter Aszites keine grosse Linderung brachte. Am Sonntag ist sie friedlich, schmerzfrei und würdig im Beisein ihrer Söhne gestorben. So hat sich ihr Wunsch erfüllt.

Noch immer hänge ich der einmaligen Begegnung mit dieser speziellen und doch so unberühmten, zierlichen Frau in Gedanken nach: Was hat diese Frau so stark gemacht? Nicht nur war ihr psychosoziales Schicksal in keiner Weise ein einfaches gewesen, auch eine Darmresektion mit vierzig Jahren, eine Tumorenukleation mit Verlust des rechten Auges, Kunstaugeneinsatz, vier Knieprothesenoperationen und eine Oberarmfraktur hatte sie erlitten. Sie hat ihr Leben lang in materiell bescheidensten Verhältnissen gelebt und trotzdem Freude am Leben gefunden.

Würde es auch andern Menschen besser gehen, ginge es ihnen "weniger gut", im Sinne der von V. Frankel postulierten noogenen Neurose, bzw. der Mensch auf der Suche nach dem Sinn? Aber, muss es denn Krieg geben, damit die Suizidraten sinken? Erkennen wir die indirekt krankmachenden Einflüsse unserer Überflussgesellschaft und wie begegnen wir Ärztinnen und Ärzte ihnen? Können Kinder stark gemacht werden, an den Schwierigkeiten des Lebens nicht zu zerbrechen, sondern in ihnen eine Herausforderung zu sehen und Lebenssinn zu finden - oder ist dies rein genetisch bedingt?

In ihrem letzten Willen zu Lebzeiten, welcher der KG beilag, schrieb sie u.a., dass an ihrer Beerdigung kein Lebenslauf verlesen werden solle. Sollte das Schwere auch nach dem Tod im Vergangenen, Vergessenen bleiben, um nicht weiter Schmerz zu verursachen, oder wollte sie gerade keine Preisung ihrer Stärke "trotz allem»? Hat sie mir deshalb so blitzlichtartig aus ihrem Leben erzählt, weil sie spürte, dass es zu Ende ging, und sie so doch noch irgendwo eine Art Lebensspur in jemandes Erinnerung liess? Ich hoffe, ihrem Willen hier nicht zuwidergehandelt zu haben. 